\title{
Temperature Dependence of the Silicon Nitride Volume Plasmon
}

\author{
Brian Zutter ${ }^{1}$, Matthew Mecklenburg ${ }^{2}$, and B. C. Regan ${ }^{1}$ \\ ${ }^{1}$ Department of Physics and Astronomy \& California NanoSystems Institute, University of California, \\ Los Angeles, CA, USA \\ 2. Center for Electron Microscopy and Microanalysis, University of Southern California, Los Angeles, \\ CA, USA
}

Silicon nitride membranes are commonly used as electron transparent windows in transmission electron microscope (TEM) in situ experiments. A pair of silicon nitride membranes, each suspended over a hole in a silicon wafer and generally 10-100 nm thick, can enclose a liquid or gas and maintain a high vacuum outside the enclosed region [1]. In addition, microelectronic devices and MEMS-based heating holders are commonly microfabricated on top of silicon nitride membrane windows. In the TEM the beam electrons primarily lose energy in a thin silicon nitride membrane by inducing a volume plasmon. Thus in many in situ TEM experiments the silicon nitride plasmon has the potential to allow, without additional sample preparation, temperature mapping across the field of view through plasmon energy expansion thermometry (PEET) [2]. However, previously the silicon nitride plasmon's temperature dependence has not been measured.

We measured the temperature dependence of the silicon nitride volume plasmon with a transmission electron microscope (JEOL JEM-2100F) operating at a high tension of 80kV, a MEMS based heating holder (DENS solutions Wildfire S3), and an electron energy loss spectrometer (Gatan Quantum SE GIF). Spectrum images of a silicon nanoparticle supported on a silicon nitride membrane were acquired for a range of temperatures. A silicon nitride plasmon energy map was made from each spectrum image by taking the energy difference between the zero-loss peak (ZLP) (Gaussian fit between $-0.45 \mathrm{eV}$ and $0.45 \mathrm{eV}$ ) and the silicon nitride volume plasmon peak (Lorentzian fit between $15.9 \mathrm{eV}$ and $26.4 \mathrm{eV}$ ). The resulting plasmon maps (with histograms) for two temperatures (of 29 acquired), $25{ }^{\circ} \mathrm{C}$ and 1250 ${ }^{\circ} \mathrm{C}$, are shown in Figure 1(A) and Figure 1(B). The shift in the plasmon energy between $25{ }^{\circ} \mathrm{C}$ and $1250^{\circ} \mathrm{C}$ was $\sim 210 \mathrm{meV}$, or about 8 pixels on the CCD of the spectrometer (set for a dispersion of 25 $\mathrm{meV} / \mathrm{channel})$.

The plasmon energy histograms can be sorted into two groups, corresponding to pixels away from and near to the aluminum nanoparticles. (While the silicon nitride plasmon is detectable in regions that also include silicon, we do not include that data here.) Each group of pixels has an approximately Gaussian distribution, as is shown by the Gaussian fits to each region. Center and width values extracted from these fits (and the 27 not shown) are plotted versus temperature in Figure 2.

The slope in the plasmon energy plot (Figure 2, left) indicates a plasmon energy change of $-0.17 \pm 0.02$ $\mathrm{meV} / \mathrm{K}$, which is substantially smaller than aluminum's value of $-0.58 \pm 0.07 \mathrm{meV} / \mathrm{K}[3,4]$. As a silicon nitride membrane is heated, its plasmon energy decreases because of thermal expansion. The standard deviation in each of the two pixel groups remains approximately constant throughout the experiment (each group is separated by $\sim 500-600 \mathrm{meV}$ in energy). Thus increasing the temperature in the silicon nitride membrane decreased each plasmon energy, but did not change the plasmon widths. The linear relation between temperature and the plasmon energy indicates that such (nearly ubiquitous) silicon nitride membranes have the potential to serve as in situ thermometers. 
References:

[1] E. R. White et al, Applied Physics Express 4 (2011), p. 055201.

[2] M Mecklenburg et al, Science 347 (2015), p. 629-632.

[3] M P Seah and G C Smith, Journal of Materials Science 21 (1986), p. 1305-1309.

[4] G Meyer, Zeitschrift fur Physik 148 (1957), p. 61-71.

[5] This work was supported in part by FAME, one of six centers of STARnet, a Semiconductor Research Corporation program sponsored by MARCO and DARPA. Data presented were acquired at the Center for Electron Microscopy and Microanalysis at the University of Southern California.
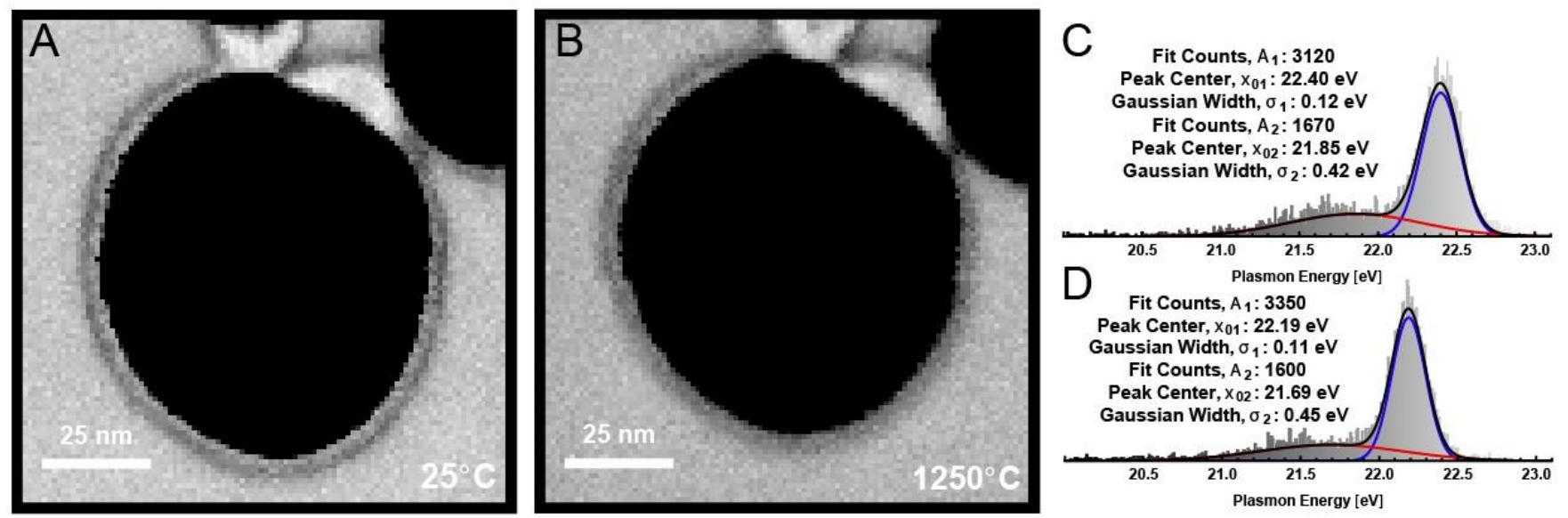

Figure 1. Plasmon energy maps acquired at $25^{\circ} \mathrm{C}(\mathrm{A})$ and $1250^{\circ} \mathrm{C}(\mathrm{B})$. A histogram of the plasmon energies in (A) is shown in (C), and likewise for (B) and (D). The histogram of plasmon energies has been fit to two Gaussian functions, and their fit values are indicated. The fit counts refers to the number of pixels in the image associated with that particular fit.
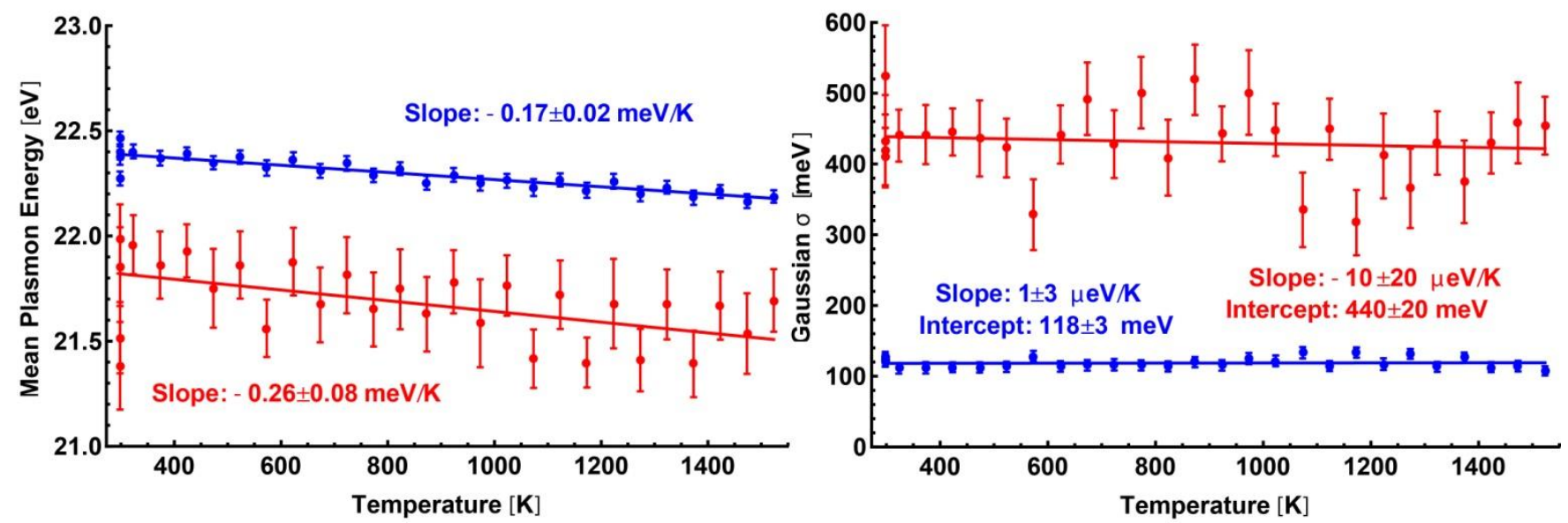

Figure 2. The measured mean plasmon energy (left) and its standard deviation (right) as a function of temperature, as determined by fits to the plasmon energy histograms. 